\title{
Isolation and complete genome sequence analysis of a novel ovine adenovirus type representing a possible new mastadenovirus species
}

\author{
Márton Z. Vidovszky ${ }^{1}\left[\right.$ D Levente Szeredi $^{2} \cdot$ Andor Doszpoly $^{1} \cdot$ Balázs Harrach $^{1} \cdot$ Ákos Hornyák $^{2}$
}

Received: 18 March 2019 / Accepted: 2 May 2019 / Published online: 31 May 2019

(c) The Author(s) 2019

\begin{abstract}
Pathological examination of a suckling male lamb showed severe viral pneumonia with suspected bacterial superinfection. Adenovirus was detected by immunohistochemical examination of the affected lung samples. Detection of the suspected adenovirus by PCR and subsequent isolation of the virus were successful. Using next-generation sequencing, the full genome of this ovine adenovirus was sequenced and analysed. A genome sequence comparison showed that it was a novel mastadenovirus type (named "ovine adenovirus 8") that did not belong to any of the established adenovirus species. The genome is 36,206 bp long, containing 93-bp inverted terminal repeats and 29 predicted genes, including the two genus-specific genes (encoding proteins $\mathrm{V}$ and IX). Ovine adenovirus 8 shows the closest relationship to ovine adenovirus 6 . These two viruses seem to merit the establishment of a novel ovine mastadenovirus species for them, for which we proposed the name "Ovine mastadenovirus C".
\end{abstract}

Adenoviruses (AdVs) are medium-sized, non-enveloped viruses with linear dsDNA genomes. AdVs have been reported in almost all known classes of vertebrates (mammals, birds, reptiles, amphibians and fish) [1-7]. Phylogenetic analysis has shown that most AdVs infecting mammals belong to the genus Mastadenovirus. Ruminants are the only mammals that have AdVs belonging to the genus Atadenovirus as well. So far, seven types of AdVs from sheep have been described [1], belonging to three distinct AdV species accepted by the International Committee on Taxonomy of Viruses (ICTV) [3]. Two species, Ovine mastadenovirus A

Handling Editor: Graciela Andrei.

The GenBank accession number of the sequence reported in this paper is MK518392.

Electronic supplementary material The online version of this article (https://doi.org/10.1007/s00705-019-04299-6) contains supplementary material, which is available to authorized users.

Márton Z. Vidovszky

vidovszky.marton@agrar.mta.hu

1 Institute for Veterinary Medical Research, Centre for Agricultural Research, Hungarian Academy of Sciences, P.O. Box 18, 1143 Budapest, Hungary

2 Veterinary Diagnostic Directorate, National Food Chain Safety Office, 1143 Budapest, Hungary and Ovine mastadenovirus $B$ belong to the genus Mastadenovirus. Ovine adenoviruses 2-5 (OAdV-2-5) and bovine adenovirus 2 (BAdV-2) belong to Ovine mastadenovirus $A$, while OAdV-1 and caprine adenovirus 2 (from goat, GAdV2) belong to Ovine mastadenovirus B. OAdV-7 and GAdV-1 belong to the species Ovine atadenovirus $D$ of the genus Atadenovirus. Only partial genome sequences of the known OAdVs belonging to the genus Mastadenovirus, mostly from the hexon gene, are available in the GenBank database [6]. The only fully sequenced OAdV genome is that of the OAdV-7 [12]. There has not been a reported isolation or even a PCR detection of a novel type of AdV in sheep since 1983.

In 2017, a pathological and histopathological examination of a suckling male lamb showed severe viral pneumonia characteristic of adenoviral infection with suspected bacterial superinfection in the Veterinary Diagnostic Directorate, National Food Chain Safety Office of Hungary. Immunohistochemical examination (IHC) demonstrated the presence of adenovirus in the cytoplasm of few enlarged inclusion-bearing bronchiolar and alveolar epithelial cells (Fig. 1). A novel type of OAdV (strain 7508) was isolated and propagated in the OA3 (ovine testicle) cell line. The virions were concentrated by ultracentrifugation, and the viral DNA was purified using the phenol/chloroform extraction method. Nextgeneration sequencing (NGS) was performed on an Illumina MiSeq platform. A total of 13,073,060 reads were generated, 


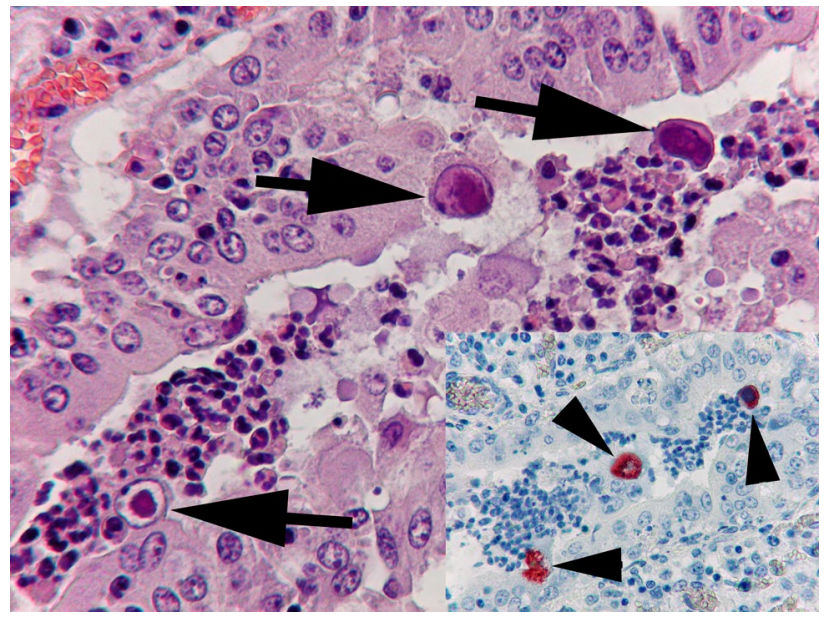

Fig. 1 Enlarged bronchiolar epithelial cells containing intranuclear inclusion bodies (arrows), and acute purulent bronchiolitis in a bronchiole (haematoxylin and eosin staining). Inset: Immunostaining of the cytoplasm of enlarged bronchiolar epithelial cells (arrowheads), showing the presence of adenoviral antigen (IHC)

with an average length of $70 \mathrm{bp}$. CLC Genomics Workbench 8.5 (CLC bio) was used for sequence assembly. Two contigs were obtained, and the gap between them was filled by PCR amplification. To determine the ends of the genome, we used direct sequencing with specific primers. The programs used for handling, identification and analysis of nucleotide sequences have been described in detail elsewhere [4]. The complete genome sequence of the novel OAdV (named "ovine adenovirus 8" [OAdV-8]) was deposited in the GenBank database under accession no. MK518392.

Here, we report the first complete genome sequence of an OAdV belonging to the genus Mastadenovirus. The genome of OAdV-8 is 36,206 bp long, and the inverted terminal repeats (ITRs) at the genome ends are $93 \mathrm{bp}$ long. The $\mathrm{G}+\mathrm{C}$ content of the whole genome proved to be unexpectedly high (70\%). OAdV-8 shows a typical mastadenovirus genome organization, containing the genus-specific genes encoding proteins $\mathrm{V}$ and IX and the E1, E3 and E4 regions. Twenty-nine genes were predicted in the OAdV-8 genome (Fig. 2), all of them showing clear homology to the genes of mastadenoviruses. During the annotation, all of the expected splicing sites were identified in the genes for E1A, IVa2, DNA-dependent DNA polymerase, pTP, and $33 \mathrm{~K}$. The gene for the whole U exon protein (UXP) was also identified by predicting splicing sites for three exons. UXP was first detected in the genome of human adenovirus 5 [10] but was later predicted also in other primate (Old World monkey) AdVs [8]. The less-conserved E4 region contains four ORFs, each showing sequence similarity (43$57 \%$ amino acid [aa] sequence identity) to the corresponding ORFs of the E4 region of bovine adenovirus 3 (BAdV-3) and deer AdV-2 [9]. The E3 region of mastadenoviruses usually contains several ORFs, and in the E3 region of OAdV-8, we identified a homologue of the BAdV-3 and deer AdV-2 E3 ORFA. This short E3 region is quite unique in ruminant AdVs, since all known E3 regions of mastadenovirus BAdVs except that of BAdV-10 are longer [11]. Nevertheless, the BAdV-3 E3 region encodes a second protein (E3 14.7K). Neither OAdV-8 nor BAdV-3 contained the E3 12.5K gene, which is present in almost every mastadenovirus [2]. On the other hand, E3 regions have not yet been sequenced from any other mastadenovirus OAdVs. This short E3 region is more typical of some rodent and bat AdVs $[4,5]$.

Sequence analysis showed that the closest relative of OAdV-8 is OAdV-6. This serotype has not been assigned to any existing ovine mastadenovirus species. For OAdV-6, only the hexon gene sequence is available in the GenBank database. This region shows $95 \%$ aa sequence identity to the corresponding region of OAdV-8. However, when only the first hexon loop sequences of the two viruses are compared, the aa sequence identity drops to $82 \%$. This part of the hexon is responsible for antigenicity, suggesting that the two viruses are distinct serotypes. Meanwhile, the high sequence similarity of the entire hexon gene implies that the two viruses may belong to the same virus species. Since the demarcation criterion for AdV species classification by the ICTV is based on the DNA-dependent DNA polymerase gene, it would be necessary at least to determine the sequence of the polymerase gene from OAdV-6 to confirm this prediction. Regardless of that status of OAdV-6, providing the complete genome sequence of a novel, distinct type of ovine mastadenovirus, we propose to designate OAdV-8 as a founding member of a novel mastadenovirus species

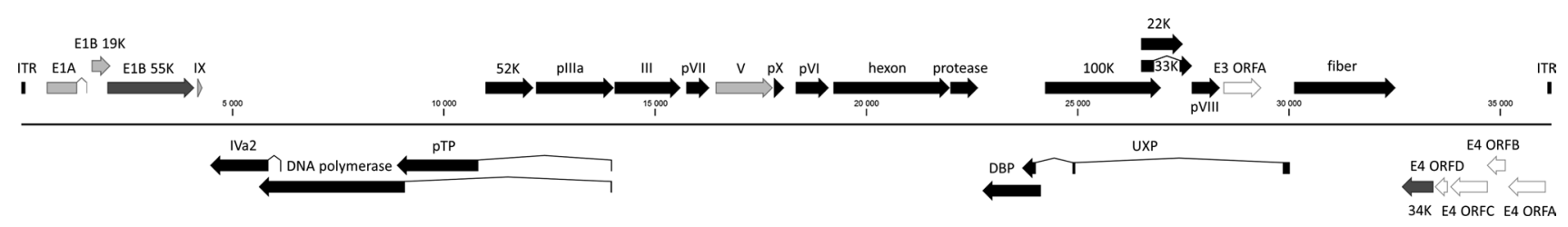

Fig. 2 Genome map of ovine adenovirus 8. A grey scale is used to indicate genes that have homologs in other adenoviruses. Black, genes present in all AdVs; dark grey, genes occurring in both mastad- enoviruses and atadenoviruses; light grey, genes specific to mastadenoviruses; white, genes with homologs only in bovine adenovirus 3 
(with the proposed name "Ovine mastadenovirus C") based on the following demarcation criteria: $36 \%$ difference in the DNA polymerase protein sequence from the most similar $\mathrm{AdV}$, a member of another species (BAdV-3, species Bovine mastadenovirus $B$; demarcation criterion, $5-15 \%$ difference), host, (sheep vs. cattle or deer), GC percentage (70\% vs. 54\% for BAdV-3), and gene content differences in the E3 region. The high GC content seems to suggest that OAdV-8 represents the original lineage that coevolved with members of the order Artiodactyla.

Acknowledgements Open access funding provided by MTA Centre for Agricultural Research (MTA ATK). This work was supported partly by a grant provided by the Hungarian Scientific Research Fund (OTKA NN128309).

\section{Compliance with ethical standards}

Conflict of interest The authors declare no conflict of interest.

Ethical approval This article does not contain any studies with live animals performed by any of the authors.

Open Access This article is distributed under the terms of the Creative Commons Attribution 4.0 International License (http://creativeco mmons.org/licenses/by/4.0/), which permits unrestricted use, distribution, and reproduction in any medium, provided you give appropriate credit to the original author(s) and the source, provide a link to the Creative Commons license, and indicate if changes were made.

\section{References}

1. Boyle DB, Pye AD, Kocherhans R, Adair BM, Vrati S, Both GW (1994) Characterisation of Australian ovine adenovirus isolates. Vet Microbiol 41:281-291

2. Davison AJ, Benkő M, Harrach B (2003) Genetic content and evolution of adenoviruses. J Gen Virol 84:2895-2908

3. Harrach B, Benkő M, Both GW, Brown M, Davison AJ, Echavaria M, Hess M, Jones MS, Kajon A et al (2011) Family Adenoviridae.
In: King AMQ, Adams MJ, Carstens EB, Lefkowitz EJ (eds) Virus taxonomy: classification and nomenclature of viruses. Ninth Report of the International Committee on Taxonomy of Viruses. San Diego, Elsevier

4. Hemmi S, Vidovszky MZ, Ruminska J, Ramelli S, Decurtins W, Greber U, Harrach B (2011) Genomic and phylogenetic analyses of murine adenovirus 2. Virus Res 160:128-135

5. Kohl C, Vidovszky MZ, Mühldorfer K, Dabrowski PW, Radonić A, Nitsche A, Wibbelt G, Kurth A, Harrach B (2012) Genome analysis of bat adenovirus 2: indications of interspecies transmission. J Virol 86:1888-1892

6. Lehmkuhl HD, Hobbs LA (2008) Serologic and hexon phylogenetic analysis of ruminant adenoviruses. Arch Virol 153:891-897

7. Podgorski II, Pantó L, Földes K, de Winter I, Jánoska M, Sós E, Chenet B, Harrach B, Benkő M (2018) Adenoviruses of the most ancient primate lineages support the theory on virus-host co-evolution. Acta Vet Hung 66:474-487

8. Podgorski II, Pantó L, Papp T, Harrach B, Benkő M (2016) Genome analysis of four Old World monkey adenoviruses supports the proposed species classification of primate adenoviruses and reveals signs of possible homologous recombination. J Gen Virol 97:1604-1614

9. Reddy PS, Idamakanti N, Zakhartchouk AN, Baxi MK, Lee JB, Pyne C, Babiuk LA, Tikoo SK (1998) Nucleotide sequence, genome organization, and transcription map of bovine adenovirus type 3. J Virol 72:1394-1402

10. Tollefson AE, Ying B, Doronin K, Sidor PD, Wold WS (2007) Identification of a new human adenovirus protein encoded by a novel late l-strand transcription unit. J Virol 81:12918-12926

11. Ursu K, Harrach B, Matiz K, Benkő M (2004) DNA sequencing and analysis of the right-hand part of the genome of the unique bovine adenovirus type 10. J Gen Virol 85:593-601

12. Vrati S, Brookes DE, Strike P, Khatri A, Boyle DB, Both GW (1996) Unique genome arrangement of an ovine adenovirus: identification of new proteins and proteinase cleavage sites. Virology 220:186-199

Publisher's Note Springer Nature remains neutral with regard to jurisdictional claims in published maps and institutional affiliations. 